\title{
Analysis of the effect of water activity on ice formation using a new thermodynamic framework
}

\author{
D. Barahona \\ Global Modeling and Assimilation Office, NASA Goddard Space Flight Center, Greenbelt, Maryland, USA
}

Correspondence to: D. Barahona (donifan.o.barahona@nasa.gov)

Received: 25 October 2013 - Published in Atmos. Chem. Phys. Discuss.: 17 January 2014

Revised: 14 May 2014 - Accepted: 21 June 2014 - Published: 30 July 2014

\begin{abstract}
In this work a new thermodynamic framework is developed and used to investigate the effect of water activity on the formation of ice within supercooled droplets. The new framework is based on a novel concept where the interface is assumed to be made of liquid molecules "trapped" by the solid matrix. It also accounts for the change in the composition of the liquid phase upon nucleation. Using this framework, new expressions are developed for the critical ice germ size and the nucleation work with explicit dependencies on temperature and water activity. However unlike previous approaches, the new model does not depend on the interfacial tension between liquid and ice. The thermodynamic framework is introduced within classical nucleation theory to study the effect of water activity on the ice nucleation rate. Comparison against experimental results shows that the new approach is able to reproduce the observed effect of water activity on the nucleation rate and the freezing temperature. It allows for the first time a phenomenological derivation of the constant shift in water activity between melting and nucleation. The new framework offers a consistent thermodynamic view of ice nucleation, simple enough to be applied in atmospheric models of cloud formation.
\end{abstract}

\section{Introduction}

Ice formation by the freezing of supercoooled droplets is an important natural and technological process. In the atmosphere it leads to the formation of cirrus and determines the freezing level of convective clouds (Pruppacher and Klett, 1997). At temperatures below $238 \mathrm{~K}$ and in the absence of ice forming nuclei, freezing proceeds by homogeneous nucleation. A significant fraction of cirrus in the upper troposphere form by this mechanism (Gettelman et al., 2012; Barahona et al., 2013). Cirrus clouds impact the radiative balance of the upper troposphere (Fu, 1996) and play a role in the transport of water vapor to the lower stratosphere (e.g., Barahona and Nenes, 2011; Jensen and Pfister, 2004; Hartmann et al., 2001). Correct parameterization of ice formation is therefore crucial for reliable climate and weather prediction (Lohmann and Feichter, 2005). Many experimental and theoretical studies have been devoted to the study of homogeneous nucleation (e.g., Kashchiev, 2000; Murray et al., 2010b; Wu et al., 2004, and references therein). Yet the role and meaning of the interfacial tension at the microscopic scale and the properties of the ice germ during the first stages of nucleation remain unclear and make the theoretical prediction of ice nucleation rates difficult.

Molecular dynamics (MD) simulations have advanced the fundamental understanding of homogeneous nucleation (e.g., Matsumoto et al., 2002; Moore and Molinero, 2011; Brukhno et al., 2008; Errington et al., 2002; Bauerecker et al., 2008). Density functional theory and direct kinetic models have also been employed (e.g., Laaksonen et al., 1995). Matsumoto et al. (2002) showed that ice nucleates when long-lived hydrogen bonds accumulate to form a compact initial nucleus. Errington et al. (2002) suggested that the formation of the initial nucleus is cooperative and only occurs when molecules accrete into clusters forming low density (LD) regions. The enthalpy of water molecules in such regions tends to resemble that of the liquid. It has been shown that the formation of $\mathrm{LD}$ regions within supercooled water is associated with an increase in the fraction of four-coordinated molecules (Moore and Molinero, 2011), 
and is thought to precede the formation of ice (Moore and Molinero, 2011; Brukhno et al., 2008; Bullock and Molinero, 2013).

MD and other detailed approaches offer a unique look at the microscopic mechanism of ice nucleation. However for climate simulations and other large scale applications, simplified and efficient descriptions of ice nucleation are required. Thus, in atmospheric modeling the theoretical study of homogeneous ice nucleation has been historically approached using the classical nucleation theory (CNT) (e.g., Khvorostyanov and Curry, 2004; Dufour and Defay, 1963; Pruppacher and Klett, 1997) and used to generate ice cloud formation parameterizations (Khvorostyanov and Curry, 2004, 2009).

CNT is often criticized due to the usage of the so-called capillary approximation, i.e., the assumption that the properties of ice clusters at nucleation are the same as those of the bulk (Kashchiev, 2000). This assumption is critical when considering the ice-liquid interfacial tension (also called specific surface energy), $\sigma_{\text {iw }}$, as CNT calculations are very sensitive to $\sigma_{\mathrm{iw}}$. Direct measurement of $\sigma_{\mathrm{iw}}$ is typically difficult and surrounded with large uncertainty (Pruppacher and Klett, 1997; Digilov, 2004). Challenges to the measurement of $\sigma_{\text {iw }}$ are related to difficulties in maintaining equilibrium between a growing ice crystal and the liquid phase at supercooled temperatures. The presence of impurities and crystal defects and the large temperature gradients near the ice-liquid interface also pose a challenge to the experimental determination of $\sigma_{\text {iw }}$ (Jones, 1974). Factors like crystal shape, type and size, and the characteristics of the ice-liquid interface may also affect the determination of $\sigma_{\text {iw }}$ (Wu et al., 2004; MacKenzie, 1997; Kashchiev, 2000).

Using independent estimates of $\sigma_{\text {iw }}$ within CNT, as for example those obtained from contact angle measurements, typically leads to large discrepancy between CNT predictions and nucleation rate measurements (MacKenzie, 1997). Thus, $\sigma_{\text {iw }}$ is often found by fitting CNT predictions to experimental measurements of the nucleation rate (e.g., Murray et al., 2010a; Khvorostyanov and Curry, 2004; MacKenzie, 1997). However $\sigma_{\text {iw }}$ obtained by this method often differs significantly from independent estimates (MacKenzie, 1997). Moreover, CNT introduces several assumptions to calculate the work of nucleation (e.g., a negligible excess of solute at the interface, a spherical ice germ, and capillarity; Kashchiev, 2000) that cannot be independently tested by obtaining $\sigma_{\mathrm{iw}}$ from nucleation rate measurements. More fundamentally, finding $\sigma_{\text {iw }}$ by fitting CNT to measured nucleation rates unties $\sigma_{\text {iw }}$ from its theoretical meaning. This may lead to inconsistencies within the theory as it is not clear what $\sigma_{\text {iw }}$ actually represents within CNT and whether it is accessible by independent methods.

Empirical correlations are most often used to describe homogeneous freezing in atmospheric models (e.g., Barahona et al., 2010; Kärcher and Lohmann, 2002; Koop et al., 2000). Experimental studies generally agree on the freezing temperature of pure water with typical variation of the order of $1 \mathrm{~K}$ (which however may represent about 2 orders of magnitude variation in nucleation rate) (Murray et al., 2010a; Pruppacher and Klett, 1997; Riechers et al., 2013). For aqueous solutions empirical correlations were often developed based on $\left(\mathrm{NH}_{4}\right)_{2} \mathrm{SO}_{4}$ and $\mathrm{H}_{2} \mathrm{SO}_{4}$ model solutions (e.g., Tabazadeh et al., 1997; Jensen et al., 1991). However Koop et al. (2000) demonstrated that when parameterized in terms of the water activity, $a_{\mathrm{w}}$, freezing temperatures become independent of the nature of the solute. Furthermore, the authors showed that when plotted in a $T-a_{\mathrm{w}}$ diagram, the melting and nucleation curves can be translated by a constant shift in water activity. This particular behavior has been confirmed in several independent studies (e.g., Zobrist et al., 2008; Knopf and Rigg, 2011; Alpert et al., 2011) and has been referred as the "water activity criteria". The Koop et al. (2000) (hereafter K00) parameterization has been incorporated in several global atmospheric models (e.g., Barahona et al., 2010; Liu et al., 2007; Lohmann and Kärcher, 2002).

The empirical model of Koop et al. (2000) suggests that a general thermodynamic formulation of ice nucleation in supercooled solutions, independent of the nature of the solute, can be achieved. Yet, such theory has been elusive. Current formulations of CNT carry a dependency on $a_{\mathrm{w}}$ and it has been suggested that CNT can explain the water activity criteria (e.g., Khvorostyanov and Curry, 2004). However by adjusting the parameters of CNT to reproduce observed nucleation rates, CNT by design reproduces the observed water activity dependency of $J_{\text {hom }}$. Thus CNT cannot independently explain the water activity criteria. In fact, Koop et al. (2000) suggested that CNT and K00 can be empirically reconciled if $\sigma_{\text {iw }}$ is allowed to vary with $a_{\mathrm{w}}$ (also shown by Alpert et al., 2011). Baker and Baker (2004) followed an alternative approach and showed that the freezing temperatures measured by K00 were consistent with the point of maximum compressibility of water. The authors derived an empirical relation between $a_{\mathrm{w}}$ and the osmotic pressure which was then used to determine freezing temperatures. The work of Baker and Baker (2004) demonstrated that the water activity criteria can be understood in terms of the compressibility of water as long as certain empirical criteria are met. Recently Bullock and Molinero (2013) assumed that low density regions in supercooled water are in equilibrium with bulk water and developed an expression for the freezing temperature of water solutions as a function of $a_{\mathrm{w}}$ that roughly agrees with the results of Koop et al. (2000). Their parameterization however depends on the enthalpy difference between the hypothetical four-coordinated liquid and pure water, which is semiempirically treated and found by fitting their MD results.

In this work a new thermodynamic framework is proposed to describe ice formation by homogeneous nucleation. The new model relies on a novel picture of the solid-liquid transition placing emphasis on entropy changes across the interface. The new thermodynamic framework is introduced 


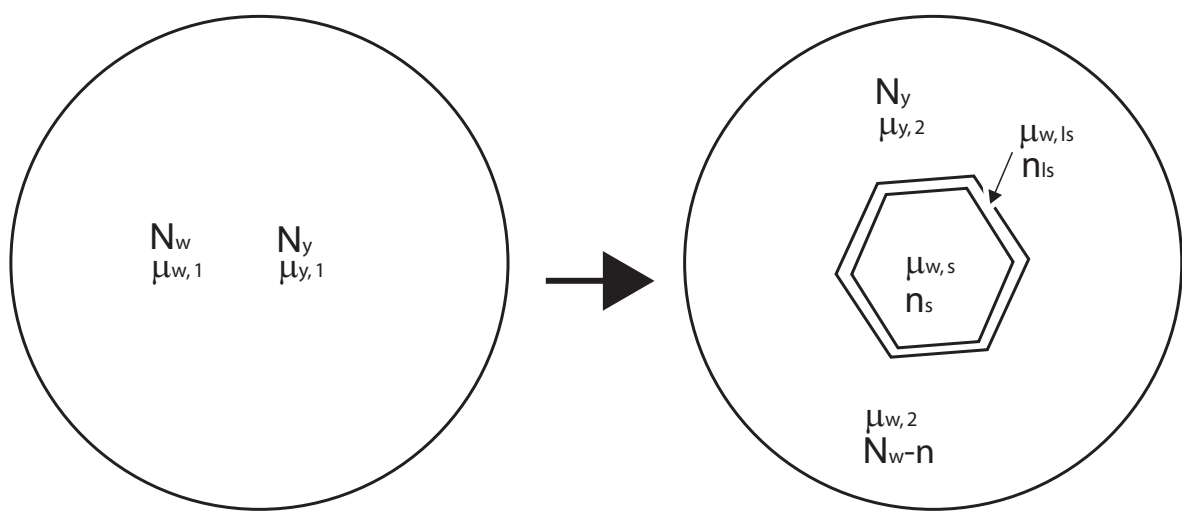

Figure 1. Scheme of the formation of an ice germ from a liquid phase. Subscripts 1 and 2 represent the state of the system before and after germ formation, respectively. $N_{\mathrm{w}}$ and $N_{y}$ represent the total molecular concentration of water and solute in the system, respectively. The subscripts ls and s refer to the liquid-solid interface and solid phases, respectively.

within CNT to study the effect of water activity on the ice nucleation rate.

\section{Theory}

Consider the system depicted in Fig. 1. The liquid droplet is assumed to be large enough so that nucleation is more likely to occur within the bulk of the liquid than at the droplet surface. The liquid is assumed to be homogeneously mixed and its cluster distribution in steady state. For simplicity it is assumed that only two components are present in solution, water (subscript, "w") and a solute (subscript, “ $y$ "), although this assumption can be easily relaxed if more than one solute is present. The Gibbs free energy of the system in stage 1 (before the formation of the ice germ) is given by

$G_{1}=N_{\mathrm{w}} \mu_{\mathrm{w}, 1}+N_{y} \mu_{y, 1}$,

where $N_{\mathrm{w}}$ and $N_{y}$ are the total number of water and solute molecules present in the liquid phase, respectively, and $\mu_{\mathrm{w}, 1}$ and $\mu_{y, 1}$ their respective chemical potentials.

After the formation of the germ (stage 2, Fig. 1) it is advantageous to consider the solid-liquid interface as a phase distinct from the bulk (Gibbs, 1957). It is assumed that no atoms of $y$ are present in the bulk of the solid phase although they may be present at the interface. However, the dividing surface is selected so that the molecular excess of solute at the interface is zero. This leads to a molecular excess of solvent at the interface and is further analyzed in Sect. 2.1. The assumption of a solute-free solid is justified on molecular dynamics simulations showing a rejection of ions into an unfrozen layer of brine away from the germ (Bauerecker et al., 2008). With this, the Gibbs free energy of the system in stage 2 is given by

$G_{2}=\left(N_{\mathrm{w}}-n_{\mathrm{s}}-n_{\mathrm{ls}}\right) \mu_{\mathrm{w}, 2}+N_{y} \mu_{y, 2}+n_{\mathrm{s}} \mu_{\mathrm{w}, \mathrm{s}}+n_{\mathrm{ls}} \mu_{\mathrm{w}, \mathrm{l}}$, where $n_{\mathrm{s}}$ and $n_{\mathrm{ls}}$ are the number of atoms in the bulk of the germ and in the interface, respectively, and $\mu_{\mathrm{w}, \mathrm{s}}$ and $\mu_{\mathrm{w}, \mathrm{s}}$, their chemical potentials. Equation (2) can be reorganized as

$G_{2}=N_{\mathrm{w}} \mu_{\mathrm{w}, 2}+N_{y} \mu_{y, 2}+n_{\mathrm{s}}\left(\mu_{\mathrm{w}, \mathrm{s}}-\mu_{\mathrm{w}, 2}\right)+n_{\mathrm{ls}}\left(\mu_{\mathrm{w}, \mathrm{ls}}-\mu_{\mathrm{w}, 2}\right)$.

Using Eqs. (1) and (3), the work of germ formation $\Delta G=G_{2}-G_{1}$ can be written as

$\Delta G=\Delta G_{\mathrm{sln}}+n_{\mathrm{s}}\left(\mu_{\mathrm{w}, \mathrm{s}}-\mu_{\mathrm{w}, 2}\right)+n_{\mathrm{ls}}\left(\mu_{\mathrm{w}, \mathrm{ls}}-\mu_{\mathrm{w}, 2}\right)$,

where $\Delta G_{\text {sln }}$ is the change in the Gibbs free energy of the bulk solution caused by the appearance of the germ, i.e.,

$\Delta G_{\text {sln }}=N_{\mathrm{w}}\left(\mu_{\mathrm{w}, 2}-\mu_{\mathrm{w}, 1}\right)+N_{y}\left(\mu_{y, 2}-\mu_{y, 1}\right)$.

Equation (4) indicates that the work of germ formation originates from (i) changes in the composition of the liquid phase, (ii) the formation of the interface and (iii) the formation of the bulk of the solid. Using the equilibrium between ice and the liquid solution as reference state, the latter can be written in the form (Kashchiev, 2000)

$\mu_{\mathrm{w}, \mathrm{s}}-\mu_{\mathrm{w}, 2}=-k_{\mathrm{B}} T \ln \left(\frac{a_{\mathrm{w}}}{a_{\mathrm{w}, \mathrm{eq}}}\right)$,

where $k_{\mathrm{B}}$ is the Boltzmann constant, $a_{\mathrm{w}, \mathrm{eq}}$ is the equilibrium water activity between bulk liquid and ice, and $a_{\mathrm{w}}$ is the activity of water in stage 2 .

The term $\Delta G_{\text {sln }}$ in Eq. (5) arises because the solute must be "unmixed" (Black, 2007) to form a solute-free germ. This causes a change in the molar composition of the liquid phase and an entropic cost to the system (Bourne and Davey, 1976). Thus, $\Delta G_{\text {sln }}$ is proportional to the mixing entropy of the system

$$
\frac{\Delta G_{\mathrm{s} \ln }}{k_{\mathrm{B}} T}=-N_{\mathrm{w}} \ln \left(\frac{a_{\mathrm{w}}}{a_{\mathrm{w}, 1}}\right)-N_{y} \ln \left(\frac{a_{y}}{a_{y, 1}}\right)-n \ln a_{\mathrm{w}},
$$


where $n=n_{\mathrm{s}}+n_{\mathrm{ls}}$ is the total number of molecules in the germ, and $a_{\mathrm{w}, 1}$ and $a_{y, 1}$ are the activities of water and solute in the initial stage (Fig. 1), respectively. If the droplet size is much larger than the ice germ, which is almost always the case for ice nucleation, then $a_{\mathrm{w}} \approx a_{\mathrm{w}, 1}$ and $a_{y} \approx a_{y, 1}$, and to a good approximation

$\Delta G_{\mathrm{s} \ln } \approx-n k_{\mathrm{B}} T \ln a_{\mathrm{w}}$.

The term $\Delta G_{\text {sln }}$ is usually neglected on the basis that the liquid phase is much larger than the ice germ (i.e., the liquid phase is considered semi-infinite with respect to the solid). However, Eq. (8) shows that although $\Delta G_{\text {sln }}$ is typically small for dilute solutions, it may become comparable to $\Delta G$ for $a_{\mathrm{w}}<1$.

\subsection{Energy of formation of the interface}

To further develop Eq. (4) it is necessary to introduce a model of the solid-liquid interface. Theoretical models suggest that the solid-liquid interface is characterized by the organization of randomly moving liquid molecules into positions determined by the solid matrix (Spaepen, 1975; Karim and Haymet, 1988; Haymet and Oxtoby, 1981). Associated with this increased order is a decrease in the partial molar entropy of the liquid molecules. Since the solid determines the positions of the molecules at the interface, the partial molar entropy at the interface must approximate the bulk entropy of the solid. However the interface molecules are liquidlike, and their enthalpy remains close to the bulk enthalpy of the liquid (Black, 2007). This is in line with the work of Reinhardt et al. (2012) who consider the molecules in the bulk ice as those with at least three connections whereas those at the surface of the solid as having only two connections but with at least one neighbor with three connections. This picture implies that the system must pay the maximum entropic cost during the formation of the germ (Spaepen, 1975; Black, 2007). The entropic nature of the thermodynamic barrier for nucleation has been confirmed by molecular dynamics simulations (Reinhardt and Doye, 2013).

Following the conceptual picture described above, the interface is assumed to be made of liquid molecules "trapped" by the solid matrix. The outermost layer of the solid along with the adjacent liquid are considered part of the interface. In reality the interface may resemble a continuous transition between solid and liquid, characterized by increasing order on the solid side (Karim and Haymet, 1988). Assuming the interface as a distinct phase creates molecular excesses of solute and solvent, which must be explicitly accounted for. This conceptual model is used below to develop an expression for the energy of formation of the interface.

The change in the partial molar free energy of water associated with the formation of the interface is given by

$\mu_{\mathrm{w}, \mathrm{ls}}-\mu_{\mathrm{w}, 2}=h_{\mathrm{w}, \mathrm{ls}}-T s_{\mathrm{w}, \mathrm{ls}}-\mu_{\mathrm{w}, 2}$, where $s_{\mathrm{w}, \mathrm{s}}$ is the entropy of the interface molecules. Assuming that the entropy of the molecules at the interface approximates the entropy of the bulk solid, i.e., $s_{\mathrm{w}, \mathrm{ls}} \approx s_{\mathrm{W}, \mathrm{s}}$, Eq. (9) can be written as

$\mu_{\mathrm{w}, \mathrm{s}}-\mu_{\mathrm{w}, 2}=h_{\mathrm{w}, \mathrm{ls}}-T s_{\mathrm{w}, \mathrm{s}}-\mu_{\mathrm{w}, 2}$.

Taking into account that $\mu_{\mathrm{w}, \mathrm{s}}=h_{\mathrm{w}, \mathrm{s}}-T s_{\mathrm{w}, \mathrm{s}}$, and using Eq. (6) into Eq. (10) we obtain

$\mu_{\mathrm{w}, \mathrm{ls}}-\mu_{\mathrm{w}, 2}=-k_{\mathrm{B}} T \ln \left(\frac{a_{\mathrm{w}}}{a_{\mathrm{w}, \mathrm{eq}}}\right)+\Delta h_{\mathrm{w}, \mathrm{ls}}$,

where $\Delta h_{\mathrm{w}, \mathrm{s}}=h_{\mathrm{w}, \mathrm{ls}}-h_{\mathrm{w}, \mathrm{s}}$ is the excess enthalpy of the water molecules at the interface.

If no solute is present, the enthalpy of the molecules at the interface approximates the enthalpy of water in the bulk, i.e., $\Delta h_{\mathrm{w}, \mathrm{s}} \approx \Delta h_{\mathrm{f}}$, being $\Delta h_{\mathrm{f}}$ the latent heat of fusion of water. However the adsorption of solute and solvent at the interface affects $\Delta h_{\mathrm{w}, \mathrm{ls}}$. Following Gibbs (1957), the effect of the molecular excess of solute and solvent on $\Delta h_{\mathrm{w}, \mathrm{ls}}$ can be written in the form (Hiemenz and Rajagopalan, 1997; Gibbs, 1957)

$\Delta h_{\mathrm{w}, \mathrm{ls}}=\Delta h_{\mathrm{f}}-\Gamma_{\mathrm{w}} k_{\mathrm{B}} T \ln a_{\mathrm{w}}-\Gamma_{y} k_{\mathrm{B}} T \ln a_{y}$,

where $\Gamma_{\mathrm{w}}$ and $\Gamma_{y}$ are the surface excess of water and solute, respectively, and represent the ratio of the number of molecules in the interface to the number of molecules at the dividing surface. $\Gamma_{\mathrm{w}}$ and $\Gamma_{y}$ depend on the position of the dividing surface (Gibbs, 1957), which is arbitrary but typically chosen so that the surface excess of solvent is zero (i.e., $\left.\Gamma_{\mathrm{w}}=0\right)$ (Kashchiev, 2000). However since $a_{\mathrm{w}}$ is typically a control variable in ice nucleation, it is convenient to choose the dividing surface as equimolecular with respect to the solute (i.e., $\Gamma_{y}=0$ ) making the surface excess a function of $a_{\mathrm{w}}$, but not of $a_{y}$. Thus, with $\Gamma_{y}=0$, Eq. (12) becomes

$\Delta h_{\mathrm{w}, \mathrm{ls}}=\Delta h_{\mathrm{f}}-\Gamma_{\mathrm{w}} k_{\mathrm{B}} T \ln a_{\mathrm{w}}$.

Equation (13) suggests that $\Delta h_{\mathrm{w}, \mathrm{ls}}$ must be independent of the nature of the solute. This can be explained as follows. Considered as a separate phase, the interface obeys the Gibbs-Duhem equation (Schay, 1976). Therefore the chemical potential of the solute, and its molecular excess at the interface, can be written in terms of the chemical potential of water, hence $a_{\mathrm{w}}$. In other words, the Gibbs-Duhem equation guarantees that the interface energy can be expressed in terms of water activity only. It follows that the dependency of $\Delta h_{\mathrm{w}, \mathrm{ls}}$ on $a_{\mathrm{w}}$ must be independent of the nature of the solute. Since $\Delta h_{\mathrm{w}, \mathrm{ls}}$ determines to great extent the nucleation rate, the dependency of $J_{\text {hom }}$ on $a_{\mathrm{w}}$ will to first order be independent of the nature of the solute.

To complete the model of the ice-liquid interface an expression for the interface thickness, hence $n_{\mathrm{ls}}$ and $\Gamma_{\mathrm{w}}$, must be derived. The number of molecules at the outermost layer 
of the solid is given by $s n^{2 / 3}$, where $s$ is a geometric constant depending on the crystal lattice (1.12 for hep crystals and 1.09 for bcc crystals; Jian et al., 2002), and $n$ is the total number of atoms in the germ. Notice that in this approximation the ice germ is allowed to have any shape, as long as it has a defined lattice structure. However the interface is likely to extend beyond the outermost layer of the solid as the solid matrix imprints some order to the adjacent liquid (Spaepen, 1975; Haymet and Oxtoby, 1981). To account for this "coverage" by the liquid on the solid, the model proposed by Spaepen (1975) is used. This model results from the explicit construction of the interface following the rules: (i) maximize the density, (ii) disallow octahedral holes and (iii) preference for tetrahedral holes (Spaepen, 1975). Using this model, Spaepen (1975) showed that there are about 1.46 molecules at the interface for each molecule in the outer layer of the solid matrix, that is, $\Gamma_{\mathrm{w}}=1.46$ and $n_{\mathrm{ls}}=\Gamma_{\mathrm{w}} s n^{2 / 3}$. Spaepens' classic model has been confirmed by experimental observations and molecular simulations (Asta et al., 2009, and references therein ). The sensitivity of $J_{\text {hom }}$ to the values of $\Gamma_{\mathrm{w}}$ and $s$ is analyzed in Sect. 3.5.

Introducing Eq. (13) into Eq. (11) we obtain

$\mu_{\mathrm{w}, \mathrm{s}}-\mu_{\mathrm{w}, 2}=-k_{\mathrm{B}} T \ln \left(\frac{a_{\mathrm{w}}}{a_{\mathrm{w}, \mathrm{eq}}}\right)+\Delta h_{\mathrm{f}}-\Gamma_{\mathrm{w}} k_{\mathrm{B}} T \ln a_{\mathrm{w}}$.

Equation (14) expresses the energy cost associated with the formation of the interface accounting for solute effects. Since it results from the consideration of the entropy reduction across the interface (i.e., negentropy production; Spaepen, 1994), this model will be referred as the negentropic nucleation framework (NNF).

\subsection{Nucleation work and nucleation rate}

Introducing Eqs. (6), (8) and (14) into Eq. (4), and rearranging we obtain

$\Delta G=-n k_{\mathrm{B}} T \ln \left(\frac{a_{\mathrm{w}}^{2}}{a_{\mathrm{w}, \mathrm{eq}}}\right)+\Gamma_{\mathrm{w}} s n^{2 / 3}\left(\Delta h_{\mathrm{f}}-\Gamma_{\mathrm{w}} k_{\mathrm{B}} T \ln a_{\mathrm{w}}\right)$

where $n=n_{\mathrm{s}}+n_{\mathrm{ls}}$ was used.

The germ size at nucleation, $n^{*}$, and the nucleation work, $\Delta G_{\text {nuc }}$, are obtained by applying the condition of mechanical equilibrium to Eq. (15), i.e.,

$$
\begin{aligned}
\frac{\mathrm{d} \Delta G_{\mathrm{nuc}}}{\mathrm{d} n *}= & -k_{\mathrm{B}} T \ln \left(\frac{a_{\mathrm{w}}^{2}}{a_{\mathrm{w}, \mathrm{eq}}}\right)+\frac{2}{3} \Gamma_{\mathrm{w}} s\left(n^{*}\right)^{-1 / 3} \\
& \left(\Delta h_{\mathrm{f}}-\Gamma_{\mathrm{w}} k_{\mathrm{B}} T \ln a_{\mathrm{w}}\right)=0 .
\end{aligned}
$$

Solving Eq. (16) for $n^{*}$ and rearranging gives

$$
n^{*}=\frac{8}{27}\left[\frac{\Gamma_{\mathrm{w}} s\left(\Delta h_{\mathrm{f}}-\Gamma_{\mathrm{w}} k_{\mathrm{B}} T \ln a_{\mathrm{w}}\right)}{k_{\mathrm{B}} T \ln \left(\frac{a_{\mathrm{w}}^{2}}{a_{\mathrm{w}, \mathrm{eq}}}\right)}\right]^{3} .
$$

The nucleation work is obtained by replacing Eq. (17) into Eq. (15). After rearranging we obtain

$\Delta G_{\text {nuc }}=\frac{4}{27} \frac{\left[\Gamma_{\mathrm{w}} s\left(\Delta h_{\mathrm{f}}-\Gamma_{\mathrm{w}} k_{\mathrm{B}} T \ln a_{\mathrm{w}}\right)\right]^{3}}{\left[k_{\mathrm{B}} T \ln \left(\frac{a_{\mathrm{w}}^{2}}{a_{\mathrm{w}, \mathrm{eq}}}\right)\right]^{2}}$.

The nucleation rate, $J_{\text {hom }}$, is given by

$J_{\text {hom }}=J_{0} \exp \left(-\frac{\Delta G_{\text {nuc }}}{k_{\mathrm{B}} T}\right)$,

where $J_{0}$ is a $T$ dependent pre-exponential factor. As in CNT, it is assumed that $J_{0}$ results from the kinetics of aggregation of single water molecules to the ice germ from an equilibrium cluster population (Kashchiev, 2000), therefore

$J_{0}=\frac{N_{\mathrm{c}} k_{\mathrm{B}} T}{h} \frac{\rho_{\mathrm{w}}}{\rho_{\mathrm{i}}} \frac{Z \Omega_{\mathrm{g}}}{v_{\mathrm{w}}} \exp \left(-\frac{\Delta G_{\mathrm{act}}}{k_{\mathrm{B}} T}\right)$,

where $N_{\mathrm{c}}$ is the number of atoms in contact with the ice germ, $\rho_{\mathrm{W}}$ and $\rho_{\mathrm{i}}$ are the bulk liquid water and ice density, respectively, $\Omega_{\mathrm{g}}$ is the germ surface area, and $\Delta G_{\text {act }}$ is the activation energy of the water molecules in the bulk of the liquid. $\Delta G_{\text {act }}$ represents the energy required for the water molecules to move from their equilibrium positions in the bulk to a new equilibrium position at the solid-liquid interface, and is closely related to the self-diffusion coefficient of water (Pruppacher and Klett, 1997). $Z$ is the Zeldovich factor, given by

$Z=\left[\frac{\Delta G_{\mathrm{nuc}}}{3 \pi k_{\mathrm{B}} T\left(n^{*}\right)^{2}}\right]^{1 / 2}$.

\subsection{Classical nucleation theory}

CNT is commonly used to describe homogeneous ice nucleation (e.g., Khvorostyanov and Curry, 2004) and is therefore important to compare the NNF model against CNT predictions. According to CNT, the work of nucleation, $\Delta G_{\mathrm{CNT}}$, is given by (Pruppacher and Klett, 1997)

$\Delta G_{\mathrm{CNT}}=\frac{16 \pi \sigma_{\mathrm{iw}}^{3} v_{\mathrm{w}}^{2}}{3\left(k_{\mathrm{B}} T \ln S_{\mathrm{i}}\right)^{2}}$,

where $S_{\mathrm{i}}=a_{\mathrm{w}}\left(p_{\mathrm{s}, \mathrm{w}} / p_{\mathrm{s}, \mathrm{i}}\right)$ is the saturation ratio with respect to the ice phase. The critical germ size is given by

$n_{\mathrm{CNT}}^{*}=\frac{32 \pi \sigma_{\mathrm{iw}}^{3} v_{\mathrm{w}}^{2}}{3\left(k_{\mathrm{B}} T \ln S_{\mathrm{i}}\right)^{3}}$.

The nucleation rate for CNT is obtained by replacing Eq. (23) into Eq. (19).

$J_{\mathrm{CNT}}=J_{0} \exp \left(-\frac{\Delta G_{\mathrm{CNT}}}{k_{\mathrm{B}} T}\right)$,

where $J_{0}$ is defined as in Eq. (20). 


\subsection{Interfacial tension}

The usage Eq. (24) requires the knowledge of $\sigma_{\text {iw }}$ which is typically found by fitting $J_{\mathrm{CNT}}$ to experimental measurements (e.g., Murray et al., 2010a; Khvorostyanov and Curry, 2004). Several empirical expressions for $\sigma_{\mathrm{iw}}$ have been developed using this approach (e.g., Pruppacher and Klett, 1997; Dufour and Defay, 1963). Here instead two new general expressions, one empirical and one theoretical, are derived to express $\sigma_{\text {iw }}$.

Attempts to derive general expressions for $\sigma_{\text {iw }}$ are often based on the approach of Turnbull (1950), who noticed that for a large number of compounds $\sigma_{\mathrm{iw}}$ was approximated by the relation

$\sigma_{\mathrm{iw}}=\frac{k_{T} \Delta h_{\mathrm{f}}}{v_{\mathrm{w}}^{2 / 3}}$,

where $k_{T}$ is an empirical constant equal to 0.32 for water. Equation (25) is mostly valid at low supercooling although it has been applied in the analysis of ice nucleation (MacKenzie, 1997). The model presented in Sect. 2 as well as the results of Koop et al. (2000), indicate that besides $T, \sigma_{\text {iw }}$ must also depend on $a_{\mathrm{w}}$, which is not captured by Eq. (25).

An independent estimate of $\sigma_{\mathrm{iw}}$, not obtained from nucleation rate measurements, can be derived from the NNF model as follows. Taking into account that the energy of formation of the interface in CNT is given by $\sigma_{\mathrm{iw}} \Omega_{\mathrm{g}}$ and using Eq. (13) we can write

$\sigma_{\mathrm{iw}} \Omega_{\mathrm{g}}=n_{\mathrm{ls}}\left(\Delta h_{\mathrm{f}}-\Gamma_{\mathrm{w}} k_{\mathrm{B}} T \ln a_{\mathrm{w}}\right)$.

Assuming a spherical ice germ and using $n_{1 \mathrm{~s}}=\Gamma_{\mathrm{w}} s n^{2 / 3}$, Eq. (26) can be solved for $\sigma_{\text {iw }}$ in the form

$\sigma_{\mathrm{iw}}=\frac{\Gamma_{\mathrm{w}} s\left(\Delta h_{\mathrm{f}}-\Gamma_{\mathrm{w}} k_{\mathrm{B}} T \ln a_{\mathrm{w}}\right)}{\left(36 \pi v_{\mathrm{w}}^{2}\right)^{1 / 3}}$.

Equation (27) provides an independent, first principles estimate of $\sigma_{\mathrm{iw}}$, obtained without the usage of nucleation rate data. It incorporates the dependency of $\sigma_{\mathrm{iw}}$ on both, $T$ and $a_{\mathrm{w}}$. For $a_{\mathrm{w}}=1$, Eq. (27) has the same form as the Turnbull (1950) expression (Eq. 25). Comparing Eqs. (27) and (25) and rearranging, we obtain for $a_{\mathrm{w}}=1$

$k_{T}=\Gamma_{\mathrm{w}} s(36 \pi)^{-1 / 3}$.

The surface area parameter, $s$, is set to $1.105 \mathrm{~mol}^{2 / 3}$, that is, the ice germ structure is assumed to lie somewhere between a bcc $\left(s=1.12 \mathrm{~mol}^{2 / 3}\right)$ and a hcp $\left(s=1.09 \mathrm{~mol}^{2 / 3}\right)$ crystal (Jian et al., 2002), justified on experimental studies showing that ice forms as a stacked disordered structure (Malkin et al., 2012). From the model of Spaepen (1975), $\Gamma_{\mathrm{w}}=1.46$ (Sect. 2.1). Using these values into Eq. (28) gives $k_{T}=0.33$, which is very close to reported values around 0.32 to 0.34 (Turnbull, 1950; Digilov, 2004). Thus, Eq. (28) helps to elucidate the meaning of $k_{T}$ in the empirical expression of Turnbull (1950): it is a measure of the thickness of the interface between the liquid and the solid.

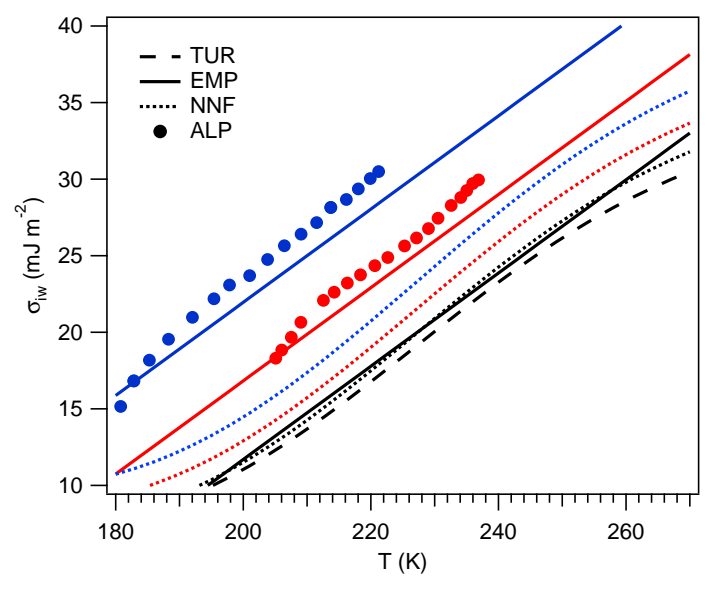

Figure 2. Interfacial tension represented by different models: TUR: correlation of Turnbull (1950), Eq. (25). EMP: empirical correlation derived from fitting CNT to the K00 parameterization (Koop and Zobrist, 2009; Koop et al., 2000), Eq. (29). NNF: theoretical expression derived from the NNF model, Eq. (27). ALP: data reproduced from the work of Alpert et al. (2011). Black, red and blue lines correspond to $a_{\mathrm{W}}$ equal to $1.0,0.9$ and 0.8 , respectively.

To explain the dependency of the interfacial tension on $a_{\mathrm{w}}$ one must consider the Gibbs model of the interface. By introducing the arbitrary dividing surface, an excess number of molecules is created around the interface between the liquid and the solid (Hiemenz and Rajagopalan, 1997). This is typically dealt with by selecting the so-called equimolecular dividing surface (EDS), in which the interface has energy but its net molecular excess is zero (Kashchiev, 2000; Schay, 1976). However the EDS cannot be defined simultaneously for the solute and the solvent. In fact, using the EDS with respect to the solvent, results in a molecular excess of solute at the interface. In Sect. 2.1 it was shown that it is advantageous to define the EDS with respect to the solute, and account explicitly for the excess of water molecules at the interface. Thus the consistency between the choice of the dividing surface and the molecular excess at the interface is explicit in NNF.

A final approach to parameterize $\sigma_{\text {iw }}$ takes advantage of the water activity criteria to derive expressions for $\sigma_{\text {iw }}$ by fitting CNT to K00. Although these expressions may depend on the specific assumptions made in implementing CNT, they would in principle be more general than other empirical approaches since the water activity criteria applies to a large number of solutes. Alpert et al. (2011) derived values for $\sigma_{\text {iw }}$ by fitting CNT to K00 and using a simplified form of the Zeldovich factor and customized expressions for $\Delta G_{\text {act }}$ (Fig. 2). Here a similar approach is followed, although based on Eq. (24) which uses a more rigorous form of Z . Also, linear dependencies of $\sigma_{\mathrm{iw}}$ on $T$ and $a_{\mathrm{w}}$ are assumed to extrapolate $\sigma_{\mathrm{iw}}$ outside of the interval where $\mathrm{K} 00$ is applicable. With this, a correlation for $\sigma_{\text {iw }}$ was obtained by fitting $J_{\mathrm{CNT}}$ (Eq. 24) to K00 in the form, 
$\sigma_{\mathrm{iw}}=0.00211-0.0513 a_{\mathrm{w}}+3.04 \times 10^{-4} T\left(\mathrm{Jm}^{-2}\right)$

with $180 \mathrm{~K}<T<273 \mathrm{~K}$ and $0.75<a_{\mathrm{w}}<1.0$. The linear dependency of $\sigma_{\mathrm{iw}}$ on $T$ and $a_{\mathrm{w}}$ is consistent with theoretical studies (Spaepen, 1994; Schay, 1976). In agreement with experimental measurements (Ketcham and Hobbs, 1969), Eq. (29) predicts $\sigma_{\text {iw }}=33.9 \mathrm{~mJ} \mathrm{~m}^{-2}$ for $T=273 \mathrm{~K}$ and $a_{\mathrm{w}}=1$ (Fig. 2).

Equations (25), (27) and (29) are selected to parameterize $\sigma_{\text {iw }}$ because they represent a progression towards incorporating additional effects of $a_{\mathrm{w}}$ within $\sigma_{\text {iw }}$. That is, Eq. (25) depends only on temperature, whereas Eq. (27) corrects for the effect of the excess of solute at the interface making $\sigma_{\text {iw }}$ a function of $a_{\mathrm{w}}$. As will be discussed in Sect. 3, the empirically derived $\sigma_{\text {iw }}$ (Eq. 29) implicitly incorporates additional effects neglected in CNT accounting for the change in the composition of the liquid phase upon nucleation (i.e., the "unmixing" energy). However, it must be emphasized that despite this progression, Eqs. (25), (27), and (29) are completely independent.

\section{Discussion}

\subsection{Interfacial tension}

The different parameterizations of $\sigma_{\text {iw }}$ presented in Sect. 2.4 are depicted in Fig. 2. As expected, $\sigma_{\text {iw }}$ obtained from the empirical correlation derived from K00 (EMP, Eq. 29) and the data reported by Alpert et al. (2011) are in good agreement, with $\sigma_{\text {iw }}$ from the latter being slightly higher. Since the same data is used in deriving both expressions (i.e., the K00 parameterization), differences between the values of $\sigma_{\mathrm{iw}}$ of Alpert et al. (2011) and Eq. (29) only result from differences in the implementation of CNT. That is, the different values of $\Delta G_{\text {act }}$ and $Z$ used in each case. The empirical correlation presented here (Eq. 29) represents the best fit between CNT and K00, with CNT as described in Sect. 2.3.

For $a_{\mathrm{w}}=1$ there is good agreement in $\sigma_{\mathrm{iw}}$ from all the models presented in Sect. 2.4. This is remarkable given that they are completely independent, derived from different nucleation rate data, or in the case of NNF completely theoretical. Still, $\sigma_{\text {iw }}$ differs by about $2 \mathrm{~mJ} \mathrm{~m}^{-2}$ which may represent up to 3 orders of magnitude difference in $J_{\text {hom }}$ (Sect. 3.2).

The NNF model predicts slightly higher $\sigma_{\text {iw }}$ than the value found by application of Eq. (25). This is because the implied constant $k_{T}$ by the NNF model is slightly higher $(0.33)$ than the value of 0.32 used by Turnbull (1950). Still, since Eq. (25) depends only on $T$, the difference between the NNF and the TUR curves for $a_{\mathrm{w}}<1$ (Fig. 2) represents the effect of $a_{\mathrm{w}}$ on $\sigma_{\mathrm{iw}}$.

For $a_{\mathrm{w}}=1$ the K00 and the NNF curves in Fig. 2 are in a good agreement. However for $a_{\mathrm{w}}<1, \sigma_{\mathrm{iw}}$ increases less steeply for the NNF-derived $\sigma_{\text {iw }}$ than suggested by the empirical correlation, Eq. (29). This difference however does not result from additional surface effects, but from an empirical correction to the assumption of a negligible change in the composition of the liquid phase upon nucleation in CNT. This can be explained as follows. Introducing the NNFderived $\sigma_{\text {iw }}$ (Eq. 27) into Eq. (22) does not make the nucleation work by NNF and CNT equal due to the quadratic dependency on $a_{\mathrm{w}}$ in the denominator of Eq. (18), which results from the additional term, $\Delta G_{\text {sln }}$, in the NNF model (Eq. 4). Removing $\Delta G_{\sin }$ from NNF would make the nucleation work by NNF and CNT equal when $\sigma_{\text {iw }}$ derived from NNF is used. Since the empirical $\sigma_{\text {iw }}$ correlation (Eq. 27) is obtained by constraining CNT to K00, and as will be shown in Sect. 3.2, $J_{\text {hom }}$ from NNF is close to K00, it follows that the empirical $\sigma_{\text {iw }}$ fit does not only parameterizes the effect of $a_{\mathrm{W}}$ on $\sigma_{\mathrm{iw}}$ but also corrects for the assumption of a negligible $\Delta G_{\text {sln }}$ in CNT. This explains the higher sensitivity of $\sigma_{\text {iw }}$ to $a_{\mathrm{w}}$ in the empirical correlation (EMP, Fig. 2) than in the NNF-derived expression.

\subsection{Nucleation rate}

Figure 3 shows the nucleation rate calculated from K00, NNF and CNT. The values used for the parameters of Eqs. (18) to (24) are listed in Table A1. The experimental results of Murray et al. (2010a) (M10) and Riechers et al. (2013) (R13) are also included in Fig. 3. Murray et al. (2010a) compared experimentally determined nucleation rates from several sources and found about a factor of 10 variation in $J_{\text {hom }}$ for pure water. Riechers et al. (2013) recently developed a new experimental technique based on microfluidics to measure $J_{\text {hom }}$. Although these correlations are only applicable around $236 \mathrm{~K}$, they are included as reference for the limiting case of $a_{\mathrm{w}}=1$.

The "freezing temperature", $T_{\mathrm{f}}$, is defined as the solution to

$J_{\text {hom }}\left(T_{\mathrm{f}}\right) \Delta t v_{\mathrm{d}}=1$,

where $\Delta t$ is the experimental timescale and $v_{\mathrm{d}}$ the droplet volume. $T_{\mathrm{f}}$ represents the temperature for which about $63 \%$ of droplets in a monodisperse droplet size distribution are frozen (or $50 \%$ in a lognormal distribution; Barahona, 2012). Defining $T_{\mathrm{f}}$ as in Eq. (30) minimizes the impact of droplet volume dispersion on $T_{\mathrm{f}}$ (Barahona, 2012). $T_{\mathrm{f}}$ is calculated by numerical iteration, assuming $\Delta t=10 \mathrm{~s}$ and a mean droplet diameter of $10 \mu \mathrm{m}$, selected to match to the conditions used by Koop et al. (2000).

There is overlap between all the curves of Fig. 3 for $T$ around $236 \mathrm{~K}$, that is, near the homogeneous freezing temperature of pure water $\left(a_{\mathrm{w}}=1\right)$ with the correlation of Riechers et al. (2013) being slightly lower than the other curves (although likely within the range of uncertainty of $J_{\text {hom }}$, Sect. 3.5). For $J_{\text {hom }}>10^{20} \mathrm{~m}^{-3} \mathrm{~s}^{-1}$, CNT-TUR predicts about two orders of magnitude higher $J_{\text {hom }}$ than CNTNNF. Such high $J_{\text {hom }}$ is however rarely encountered at atmospheric conditions. The agreement between CNT-EMP 

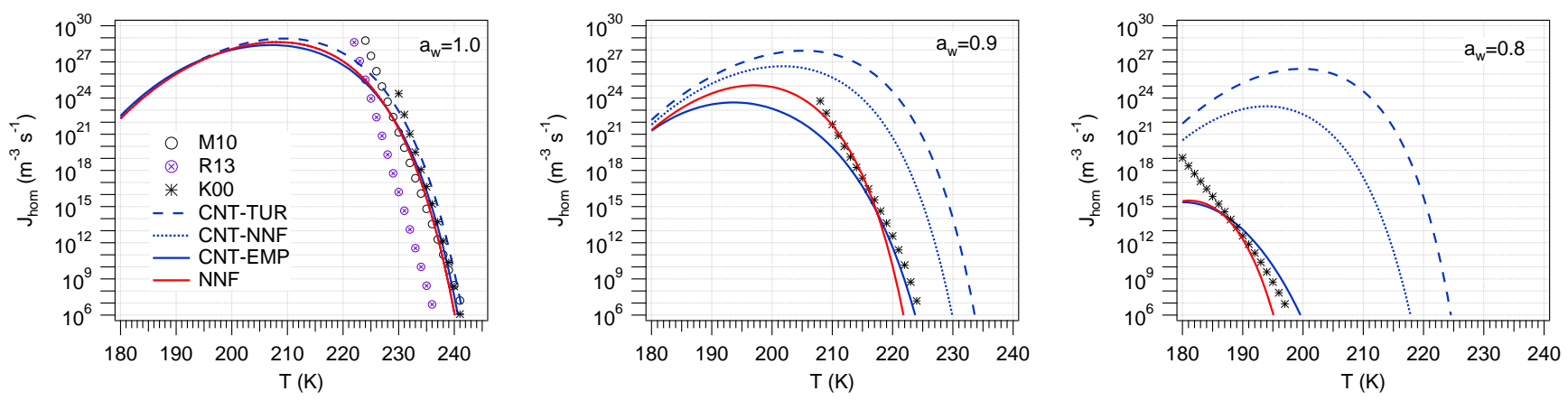

Figure 3. Homogeneous nucleation rate. K00, M10 and R13 refer to results obtained using the correlations of Koop et al. (2000), Murray et al. (2010a) and Riechers et al. (2013), respectively. For CNT $\sigma_{\text {iw }}$ was calculated using the Turnbull (1950) correlation (CNT-TUR, Eq. 25), an empirical correlation derived from fitting CNT to the K00 parameterization (CNT-EMP, Eq. 29), and a theoretical expression derived from the NNF model (CNT-NNF, Eq. 27). Results using the NNF model (Eq. 19) are also shown.

and $\mathrm{K} 00$ is by design since $\mathrm{K} 00$ data was used to develop Eq. (29), however for $J_{\text {hom }}>10^{15} \mathrm{~m}^{-3} \mathrm{~s}^{-1}$ CNT-EMP tends to predict lower $J_{\text {hom }}$ than $\mathrm{K} 00$ and NNF which results from the linear extrapolation assumed in $\sigma_{\text {iw }}$ (Sect. 2.4).

There is in general good agreement in $J_{\text {hom }}$ predicted by the NNF and the K00 models (Fig. 3). Since no data from K00 (or any other nucleation rate measurements) were used in the development of NNF, comparison against K00 constitutes an independent test of the NNF model and shows its capacity to explain observed nucleation rates. For $a_{\mathrm{w}}<1$, $\mathrm{NNF}$ and K00 agree within the typical scatter of experimentally determined $J_{\text {hom }}$ (e.g., Murray et al., 2010 b; Alpert et al., 2011). However for $a_{\mathrm{w}} \approx 0.8, \mathrm{NNF}$ seems to underpredict $J_{\text {hom }}$ by about 3 orders of magnitude, particularly for $J_{\text {hom }}<10^{10} \mathrm{~m}^{-3} \mathrm{~s}^{-1}$.

CNT and NNF show an initial increase in $J_{\text {hom }}$ as $T$ decreases, however this tendency reverses at low $T$, i.e., they predict a maximum in $J_{\text {hom }}$ when measured at constant $a_{\mathrm{w}}$. This behavior is caused by an increase in $\Delta G_{\text {act }}$ as $T$ decreases, as the role of activation of water molecules becomes increasingly more significant at low $T$ limiting $J_{\text {hom }}$ (Sect. 3.4). For $a_{\mathrm{w}}>0.9, J_{\text {hom }}$ peaks at values greater than $10^{20} \mathrm{~m}^{-3} \mathrm{~s}^{-1}$. Such high $J_{\text {hom }}$ may be difficult to measure experimentally. However for $a_{\mathrm{w}} \approx 0.8, J_{\text {hom }}$ peaks around $10^{15} \mathrm{~m}^{-3} \mathrm{~s}^{-1}$, typically found in small droplets at low $T$, and may be more accessible to experiment. The existence of a maximum in $J_{\text {hom }}$ also implies that around its peak value $J_{\text {hom }}$ is relatively insensitive to $T$. Thus around the maximum $J_{\text {hom }}$, measured freezing temperatures would be very sensitive to small changes in droplet size and cooling rate. The existence of a maximum in $J_{\text {hom }}$ is however a theoretical result and more research may be needed to elucidate its nature.

The expressions used for $\sigma_{\text {iw }}$ within CNT progressively account for additional effects of $a_{\mathrm{w}}$ on $J_{\text {hom }}$ (Sect. 2.4). Thus the impact of $a_{\mathrm{w}}$ on $J_{\text {hom }}$ through surface excess effects is represented by the difference between the CNTNNF and the CNT-TUR curves in Fig. 3 (middle and right panels). Similarly, the difference between the CNT-EMP and the CNT-NNF curves corresponds to the additional empirical correction required in $\sigma_{\mathrm{iw}}$ to account for the energy cost of making a solute-free germ, neglected in CNT (Eq. 8). Both effects imply an additional burden to $\Delta G_{\text {nuc }}$ and dramatically decrease $J_{\text {hom }}$. As $a_{\mathrm{w}}$ decreases, mixing effects tend to be more significant representing a decrease of more than 10 orders of magnitude in $J_{\mathrm{hom}}$.

Figure 4 shows that there is a wide variation in $\frac{\partial J_{\text {hom }}}{\partial a_{\mathrm{w}}}$ at constant $T$ between CNT, NNF and K00 around the freezing line (defined as in Eq. 30), even at $a_{\mathrm{w}}=1$ where Fig. 3 (left panel) shows relatively good agreement in $J_{\text {hom }}$. This is significant since $\frac{\partial J_{\text {hom }}}{\partial a_{\mathrm{w}}}$ determines to great extent the germ size (Sect. 3.3). $J_{\text {hom }}$ from the NNF model seems to decrease slightly more steeply with $a_{\mathrm{w}}$ than K00, although the agreement is within the models' uncertainty. Again, this represents an independent test of the validity of the NNF model. The agreement between CNT-EMP and K00 is by design with some deviation beyond the range of applicability of K00. $J_{\text {hom }}$ is much less sensitive to $a_{\mathrm{w}}$ for the CNT-TUR and CNTNNF curves than for the other models, particularly at low $T$, indicating the strong impact of solute surface excess and mixing effects on $J_{\text {hom }}$.

\subsection{Critical germ size}

Figure 5 shows the critical germ size in terms of the number of water molecules in the germ, calculated using NNF, CNT, and derived from the K00 expression. For the later, the nucleation theorem (Kashchiev, 2000) allows to determine $n^{*}$ directly from experimental measurements in the form

$n^{*}=-\frac{\mathrm{d} \Delta G_{\mathrm{nuc}}}{\mathrm{d} \Delta \mu_{\mathrm{w}}}+\frac{\partial \Phi}{\partial \Delta \mu_{\mathrm{w}}}$,

where $\Phi$ is the energy of formation of the interface, and $\Delta \mu_{\mathrm{w}}=-k_{\mathrm{B}} T \ln \left(\frac{a_{\mathrm{w}}}{a_{\mathrm{w}, \mathrm{eq}}}\right)$. Equation (31) can be rewritten as (Kashchiev, 2000) 

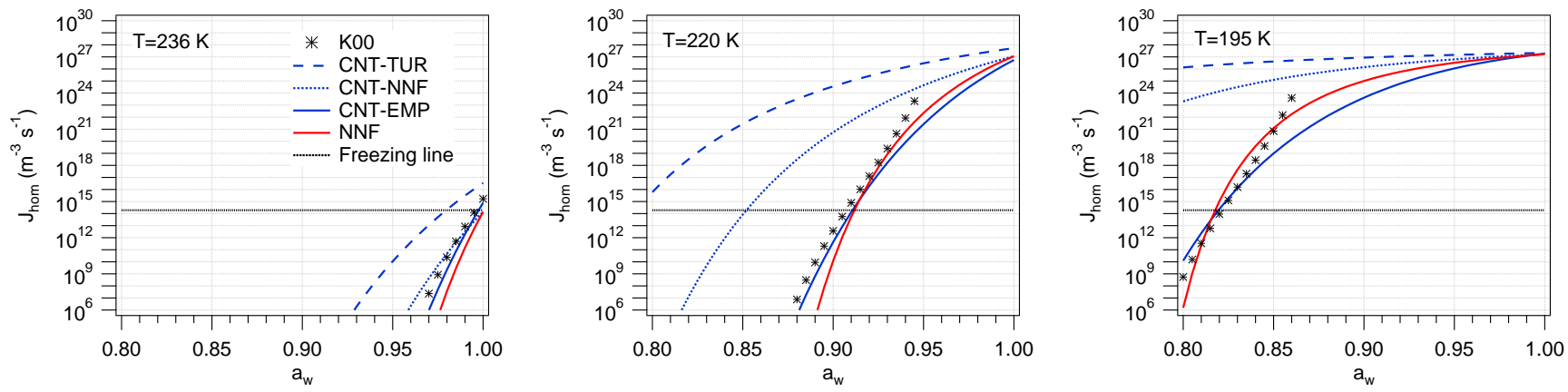

Figure 4. Homogeneous nucleation rate. K00 and NNF correspond to $J_{\text {hom }}$ obtained using the correlations of Koop et al. (2000) and the NNF model (Eq. 19) respectively. For CNT $\sigma_{\mathrm{iw}}$ was calculated using the Turnbull (1950) correlation (CNT-TUR, Eq. 25), an empirical correlation derived from fitting CNT to the K00 parameterization (CNT-EMP, Eq. 29), and a theoretical expression derived from the NNF model (CNT-NNF, Eq. 27).

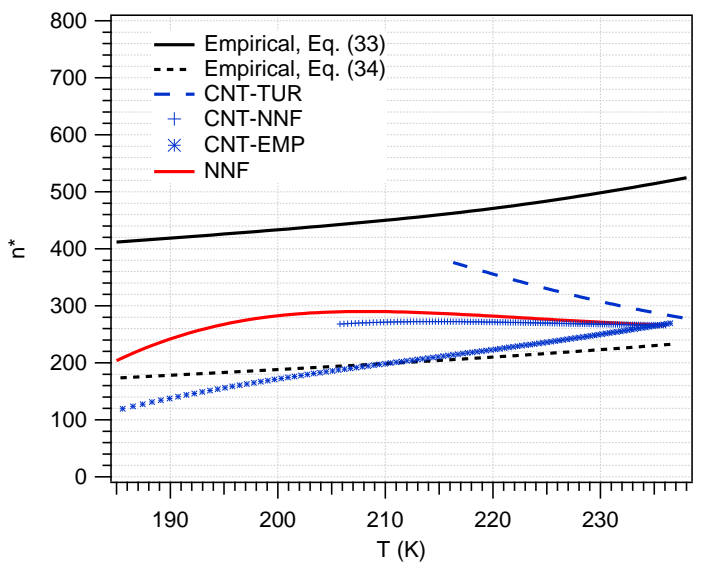

Figure 5. Critical germ size, $n^{*}$ calculated at $T_{\mathrm{f}}$ with $D_{p}=10 \mu \mathrm{m}$ and $\Delta t=10 \mathrm{~s}$. Lines labeled as empirical were obtained using the K00 correlation and a form of the nucleation theorem (Kashchiev, 2000). CNT results were obtained using $\sigma_{\text {iw }}$ from the Turnbull (1950) correlation, (CNT-TUR, Eq. 25), an empirical correlation derived from fitting CNT to the K00 parameterization (CNT-EMP, Eq. 29), and a theoretical expression derived from the NNF model (CNT-NNF, Eq. 27). Results using the NNF model (Eq. 17) are also shown.

$n^{*}=\frac{\mathrm{d} \ln J_{\mathrm{hom}}}{\operatorname{d} \ln a_{\mathrm{w}}}-1+\frac{1}{k_{\mathrm{B}} T} \frac{\partial \Phi}{\partial \ln a_{\mathrm{w}}}$.

Equation (32) is typically used assuming that $\Phi$ does not depend on $a_{\mathrm{w}}$ (Ford, 2001; Kashchiev, 2000), i.e.,

$n^{*}=\frac{\mathrm{d} \ln J_{\mathrm{hom}}}{\mathrm{d} \ln a_{\mathrm{w}}}-1$.

Using Eq. (33), along with the K00 parameterization results in $n^{*}$ between 400 and 600 molecules for $T$ between 190 and $236 \mathrm{~K}$ (Fig. 5). On the other hand, using CNT with $\sigma_{\text {iw }}$ derived from a fit to K00 (Eq. 29) results in $n^{*}$ between 100 and 250 (Fig. 5, CNT-EMP). A similar discrepancy between
K00 and CNT was found by Ford (2001) who ascribed it to limitations in CNT in describing the surface energy excess. Ford (2001) however did not account for the dependency of $\sigma_{\text {iw }}$ on $a_{\mathrm{w}}$.

From Sects. 2.4 and 3.2 it is clear that the energy of formation of the interface is not independent of $a_{\mathrm{w}}$ and may affect $n^{*}$. Using the assumption of CNT that $\Phi=\sigma_{\mathrm{iw}} \Omega_{\mathrm{g}}$ and introducing Eq. (29) into Eq. (32), we obtain for a spherical ice germ

$n^{*}=\frac{\mathrm{d} \ln J_{\mathrm{hom}}}{\mathrm{d} \ln a_{\mathrm{w}}}-1+\left(n^{*}\right)^{2 / 3} \frac{(36 \pi)^{1 / 3} v_{\mathrm{w}}^{2 / 3} a_{\mathrm{w}}}{0.0513 k_{\mathrm{B}} T}$.

Solving Eq. (34) iteratively results in $n^{*}$ around 200 for $T$ between 180 and $240 \mathrm{~K}$ (Fig. 5). This value is much lower than implied by Eq. (33) and in better agreement with CNTEMP. Thus most of the discrepancy in $n^{*}$ between CNT and Eq. (33) results from neglecting the dependency of $\Phi$ on $a_{\mathrm{w}}$. This implies that $\frac{\partial \Phi}{\partial \Delta \mu_{\mathrm{w}}}$ is not negligible and Eq. (32) instead of Eq. (33), must be used in the analysis of ice nucleation data.

The NNF model (Eq. 17) predicts $n^{*}$ around 260 for $T$ between 180 and $240 \mathrm{~K}$ (Fig. 5, line NNF). This value is slightly higher than obtained using Eq. (34). However the empirical correlation derived for $\sigma_{\text {iw }}$ (Eq. 29) used in Eq. (34) does not only account for surface effects but also corrects for neglecting $\Delta G_{\text {sln }}$ in CNT. Thus it is likely that Eq. (34) overestimates $\frac{\partial \Phi}{\partial \ln a_{\mathrm{w}}}$, even though $J_{\text {hom }}$ predicted by CNT-EMP is in agreement with K00. The slight increase in $n^{*}$ as temperature decreases predicted by NNF results from a faster decrease in the interfacial term than in the thermodynamic term of Eq. (18).

It must be noticed that $n^{*}$ shown in Fig. 5 is calculated at $T=T_{\mathrm{f}}$, which implies that $a_{\mathrm{w}}$ is not constant but varies with $T_{\mathrm{f}}$. For $T_{\mathrm{f}}>210 \mathrm{~K}$ the CNT-NNF and NNF curves in Fig. 5 remain close. However, due to the lower sensitivity of $\sigma_{\text {iw }}$ to $a_{\mathrm{w}}$ in CNT-NNF than in NNF, $T_{\mathrm{f}}$ remains above $210 \mathrm{~K}$ in the former (Fig. 6). CNT-TUR shows a strong increase in $n^{*}$ 


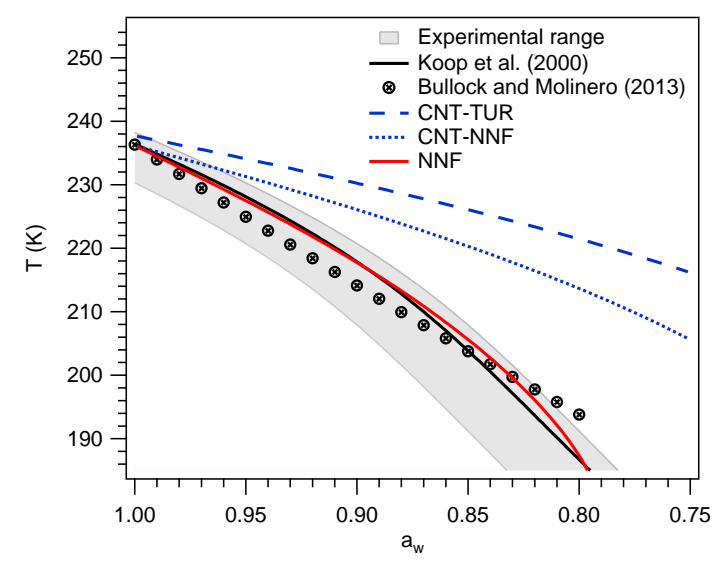

Figure 6. Freezing temperature for homogeneous nucleation. $T_{\mathrm{f}}$ was found by application of Eq. (30) assuming $D_{p}=10 \mu \mathrm{m}$ and $\Delta t=10 \mathrm{~s}$. Lines CNT-TUR and CNT-NNF and CNT-EMP correspond to classical nucleation theory using $\sigma_{\mathrm{iw}}$ from Eqs. (25), (27) and (29), respectively. $T_{\mathrm{f}}$ obtained using the K00 parameterization (Koop et al., 2000; Koop and Zobrist, 2009), the NNF model, and the correlation of Bullock and Molinero (2013) are also shown. The experimental range represents $\Delta a_{\mathrm{W}}=0.313 \pm 0.025$ (Koop and Zobrist, 2009; Alpert et al., 2011).

as $T_{\mathrm{f}}$ decreases, similarly to the behavior observed by Ford (2001).

It is also important to test whether the picture presented in Sect. 2.1 is physically plausible. The pressure change across the interface can be calculated using the generalized Laplace equation (Kashchiev, 2000),

$\Delta P=\frac{1}{v_{\mathrm{w}}} \frac{\partial \Phi}{\partial n^{*}}$,

where the solid is assumed incompressible. Direct application of Eq. (35) is somehow difficult because $n^{*}$ is not independent of $a_{\mathrm{w}}$. However for $a_{\mathrm{w}}=1, n^{*}$ can be approximated as only dependent on $T$. Thus, making $\Phi=\left(\mu_{\mathrm{w}, \mathrm{ls}}-\mu_{\mathrm{w}, 2}\right) n_{\mathrm{ls}}$ and replacing Eq. (14) into Eq. (35) we obtain for $a_{\mathrm{w}}=1$,

$\Delta P\left(a_{\mathrm{w}}=1\right)=\frac{2}{3} \frac{\Gamma_{\mathrm{w}} s \Delta h_{\mathrm{f}}}{v_{\mathrm{w}}\left(n^{*}\right)^{1 / 3}}$.

Using the parameters of Table $\mathrm{A} 1, \Delta P=336 \mathrm{bar}$ for $n^{*}=260$. This value is below the compressibility limit of water (Baker and Baker, 2004). Thus, for atmospheric conditions the increased pressure at the interface will not result in destabilization of the water structure. This indicates that the picture of the interface presented here is physically plausible. $\Delta P$ is of the same order as the osmotic pressure defined by Baker and Baker (2004), however the relation between $\Delta P$ and the osmotic pressure is not clear.

\subsection{Freezing temperature}

In this section we investigate whether the model presented in Sect. 2 is able to explain the water activity criteria of Koop et al. (2000), that is, whether the NNF model is able to independently predict a constant difference between $a_{\mathrm{w}}$ and $a_{\mathrm{w}, \text { eq }}$ when calculated at $T_{\mathrm{f}}$. Figure 6 shows $T_{\mathrm{f}}$ (Eq. 30), calculated using K00, CNT and NNF. Results using the correlation of Bullock and Molinero (2013) (hereafter BM13) derived from MD simulations are also included. The gray area in Fig. 6 represents experimental uncertainty and was obtained by setting $\Delta a_{\mathrm{w}}=a_{\mathrm{w}}-a_{\mathrm{w} \text {, eq }}=0.313 \pm 0.025$, which is the typical range of $\Delta a_{\mathrm{w}}$ found in experimental observations (Koop and Zobrist, 2009; Alpert et al., 2011; Knopf and Rigg, 2011).

Using K00 directly into Eq. (30) and finding $\Delta a_{\mathrm{w}}$ and $T_{\mathrm{f}}$ iteratively, results in an average $\Delta a_{\mathrm{w}}$ of about 0.302 for $238 \mathrm{~K}>T_{\mathrm{f}}>180 \mathrm{~K}$. The slightly lower $\Delta a_{\mathrm{w}}$ than reported by Koop and Zobrist (2009) $\left(\Delta a_{\mathrm{w}}=0.313\right)$ results from using a fixed droplet size of $10 \mu \mathrm{m}$ whereas in Koop et al. (2000) $D_{p}$ varied between 1 and $10 \mu \mathrm{m}$. Carrying out the same exercise with $J_{\text {hom }}$ derived from the NNF model results in overlap of $T_{\mathrm{f}}$ between K00 and NNF down to $190 \mathrm{~K}$ (Fig. 6). This shows that the NNF model is able to reproduce the water activity criteria and constitutes an independent theoretical derivation of the results of Koop et al. (2000).

BM13 agrees with K00 and NNF within experimental uncertainty for $T_{\mathrm{f}}$ between 200 and $233 \mathrm{~K}$, but it tends to overpredict $T_{\mathrm{f}}$ for lower temperature. This overprediction was also observed by Bullock and Molinero (2013) and was ascribed to the temperature dependency of the water activity coefficient.

Figure 6 also shows $T_{\mathrm{f}}$ calculated with CNT using the different approximations to $\sigma_{\mathrm{iw}}$ presented in Sect. 2.4. The CNT-EMP line has been omitted as by design it overlaps with the K00 line. As discussed in Sect. 3.2, the difference between the CNT-NNF and CNT-TUR curves represents the effect of the surface excess of solute on $J_{\text {hom }}$, hence $T_{\mathrm{f}}$. This effect results in about $10 \mathrm{~K}$ lower $T_{\mathrm{f}}$ than when $\sigma_{\mathrm{iw}}$ is assumed independent of $a_{\mathrm{w}}$ (curve CNT-TUR). Mixing effects, represented by the difference between the CNT-NNF and the K00 curves, become increasingly significant at low $T$ and represent about $20 \mathrm{~K}$ decrease in $T_{\mathrm{f}}$ for $a_{\mathrm{w}} \approx 0.8$.

The NNF model allows to further explore the origin of the constant shift in water activity observed by Koop et al. (2000). Using Eq. (19) into Eq. (30), and rearranging gives,

$k_{\mathrm{B}} T \ln \left(J_{0} \Delta t v_{\mathrm{d}}\right)-\frac{4}{27} \frac{\left\{\Gamma_{\mathrm{w}} s\left[\Delta h_{\mathrm{f}}-\Gamma_{\mathrm{w}} k_{\mathrm{B}} T \ln \left(a_{\mathrm{w}, \mathrm{eq}}+\Delta a_{\mathrm{w}}\right)\right]\right\}^{3}}{\left\{k_{\mathrm{B}} T \ln \left[\frac{\left(a_{\mathrm{w}, \mathrm{eq}}+\Delta a_{\mathrm{w}}\right)^{2}}{a_{\mathrm{w}, \mathrm{eq}}}\right]\right\}^{2}}=0$.

Since solutions Eq. (37) are also solutions to Eq. (30), Eq. (37) determines $T_{\mathrm{f}}$ and $\Delta a_{\mathrm{w}}$. Because of this, the left hand side of Eq. (37) is termed the characteristic freezing function.

Inspection of Eq. (37) shows that the characteristic freezing function depends only on $T$, where $\Delta a_{\mathrm{w}}$ acts a parameter defining its roots. By exploring the parameter space of Eq. (37) we can determine what values of $\Delta a_{\mathrm{w}}$ allow for real solutions to Eq. (37). This is shown in Fig. 7, where $T_{\mathrm{f}}$ is defined at the intersection between the characteristic freezing 
function and the horizontal axis. Figure 7 shows that Eq. (37) only has real solutions over a very narrow set of values of $\Delta a_{\mathrm{w}}$, i.e., $0.298<\Delta a_{\mathrm{w}}<0.306$. In other words, for $T_{\mathrm{f}}$ to exist, $\Delta a_{\mathrm{W}}$ must be almost constant between 180 and $240 \mathrm{~K}$. This explains the water activity criteria since the variation in $\Delta a_{\mathrm{w}}$ shown in Fig. 7 is well within experimental uncertainty (Fig. 6). An interesting feature of the characteristic freezing function is that it produces similar $T-a_{\mathrm{w}}$ curves for different $\Delta a_{\mathrm{w}}$ values. This means that the multiple roots of Eq. (37) are located at similar $T_{\mathrm{f}}$ for different values of $\Delta a_{\mathrm{w}}$, and always fall on the same curve (Fig. 6). The oscillating behavior of the freezing function results from the relative variation in the temperature derivative of the interfacial and thermodynamic terms defining the nucleation work (Eq. 18).

Figure 7 shows that Eq. (37) constitutes a theoretical derivation of the water activity criteria. $\Delta a_{\mathrm{w}}$ can be obtained by numerically solving Eq. (37). However for $a_{\mathrm{w}}=1$, Eq. (37) is simplified and $\Delta a_{\mathrm{w}}$ can be found by direct analytical solution, in the form,

$\Delta a_{\mathrm{w}}=1-\exp \left[-\frac{2}{3 \sqrt{3 \ln \left(J_{0} \Delta t v_{\mathrm{d}}\right)}}\left(\frac{\Gamma_{\mathrm{w}} s h_{\mathrm{f}}}{k_{\mathrm{B}} T^{*}}\right)^{3 / 2}\right]=0.304$

where $T^{*}=236.03$ is the freezing temperature at $a_{\mathrm{w}}=1$. The value of $\Delta a_{\mathrm{w}}$ in Eq. (38) was obtained using the parameters of Table A1 calculated at $T^{*} . \Delta a_{\mathrm{w}}$ is very close to the experimental value of 0.302 found by application of K00 (Fig. 6) and within experimental uncertainty of reported values (e.g., Koop and Zobrist, 2009; Alpert et al., 2011). For $T>190 \mathrm{~K}$, $\Delta a_{\mathrm{w}}$ calculated from Eq. (37) is fairly constant (being 0.300 at $T=190 \mathrm{~K}$ ). For $T<190$ there is a slight increase in $\Delta a_{\mathrm{w}}$ reaching about 0.31 at $T=180 \mathrm{~K}$. This increase is due to the increase in $\Delta G_{\text {act }}$ at low $T$.

From the agreement of BM13 with K00 (Fig. 6) Bullock and Molinero (2013) concluded that the formation of fourcoordinated water controls $T_{\mathrm{f}}$, which implies a kinetic control for nucleation. This view can be reconciled with the thermodynamic framework presented here by taking into account the role of $\Delta G_{\text {act }}$ in determining $J_{\text {hom. The prod- }}$ uct $\frac{N_{\mathrm{c}} k_{\mathrm{B}} T}{h} \frac{\rho_{\mathrm{W}}}{\rho_{\mathrm{i}}} \frac{Z \Omega_{\mathrm{g}}}{v_{\mathrm{w}}}$ in Eq. (20) is almost constant between 180 and $236 \mathrm{~K}$. Therefore the flux of molecules to the germ is controlled by $\Delta G_{\text {act }}$. In fact, introducing Eq. (18) into Eq. (19) and then into Eq. (30), we obtain after rearranging,

$\frac{\Delta G_{\mathrm{nuc}}+\Delta G_{\mathrm{act}}}{T_{\mathrm{f}}} \approx$ constant.

Equation (39) implies that an increase $\Delta G_{\text {act }}$ is balanced by a decrease in $\Delta G_{\text {nuc }}$, i.e., the increase in the driving force for nucleation at low $T$ balances the decrease in the mobility of water molecules. One can hypothesize that the formation of low density patches of water within a supercooled droplet becomes less frequent at low $a_{\mathrm{w}}$ (hence low $T_{\mathrm{f}}$ ), which translates into a larger $\Delta G_{\text {act }}$. Hence $\Delta G_{\text {act }}$ exerts a kinetic control on $T_{\mathrm{f}}$ and $\Delta G_{\text {nuc }}$ responds accordingly (Eq. 39). In other

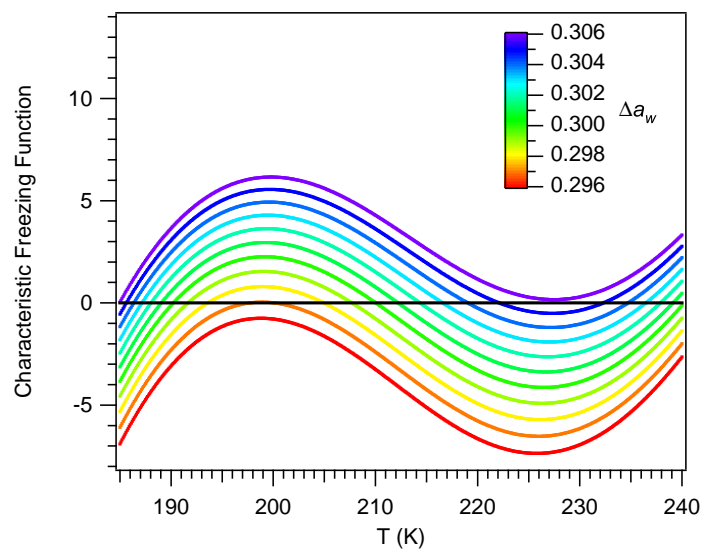

Figure 7. Characteristic freezing function for $D_{p}=10 \mu \mathrm{m}$ and $\Delta t=10 \mathrm{~s}$.

words, a kinetic constraint to nucleation implies a thermodynamic one (and vice versa), and $T_{\mathrm{f}}$ represents the temperature at which they balance. $\Delta G_{\text {act }}$ is closely related to the self-diffusivity of water (Pruppacher and Klett, 1997) and it follows that diffusivity must play a critical role in determining $J_{\text {hom }}$ at low $T$. Since $\Delta G_{\text {nuc }}$ can be defined over a purely thermodynamic basis (Sect. 2), Eq. (39) suggests that $\Delta G_{\text {act }}$ may also admit a thermodynamic description.

\subsection{Sources of uncertainty}

Besides the physical properties of water the NNF model depends on two constants: the surface coverage, $\Gamma_{\mathrm{w}}$, and the geometric constant defining the crystal lattice, $s$. It is clear that variation in physical properties, particularly the heat of fusion, will affect $J_{\text {hom }}$. The parameterization of $\Delta G_{\text {act }}$, here assumed that of pure water, would also have an effect on nucleation rates particularly at low $T$ (Pruppacher and Klett, 1997). The physical properties of water can be obtained by independent methods and it is out of the scope of this work to evaluate their accuracy.

Since they are elevated to the third power in the work of nucleation, $J_{\text {hom }}$ is very sensitive to $\Gamma_{\mathrm{w}}$ and $s$. In principle their variation would have a similar effect on the nucleation rate as variation in $\sigma_{\mathrm{iw}}$ in CNT. However $\Gamma_{\mathrm{w}}$ and $s$ can be constrained independently without using nucleation rate measurements. Furthermore, their plausible range of variation is well-constrained by the underlying physics. Variation in $\Gamma_{\mathrm{w}}$ may originate from crystal defects in the germ, and from significant order beyond the second interfacial layer. The former may be rare since defects will be energetically unfavored. The latter is more difficult to assess, however the percentage of molecules that would display order beyond the second layer is expected to be small. From Spaepen (1975)'s model, $\Gamma_{\mathrm{w}}$ is expected to be close to 1.46 since order is rapidly lost when moving from the interface into the bulk of the liquid. Assuming that $10 \%$ of the third layer molecules 

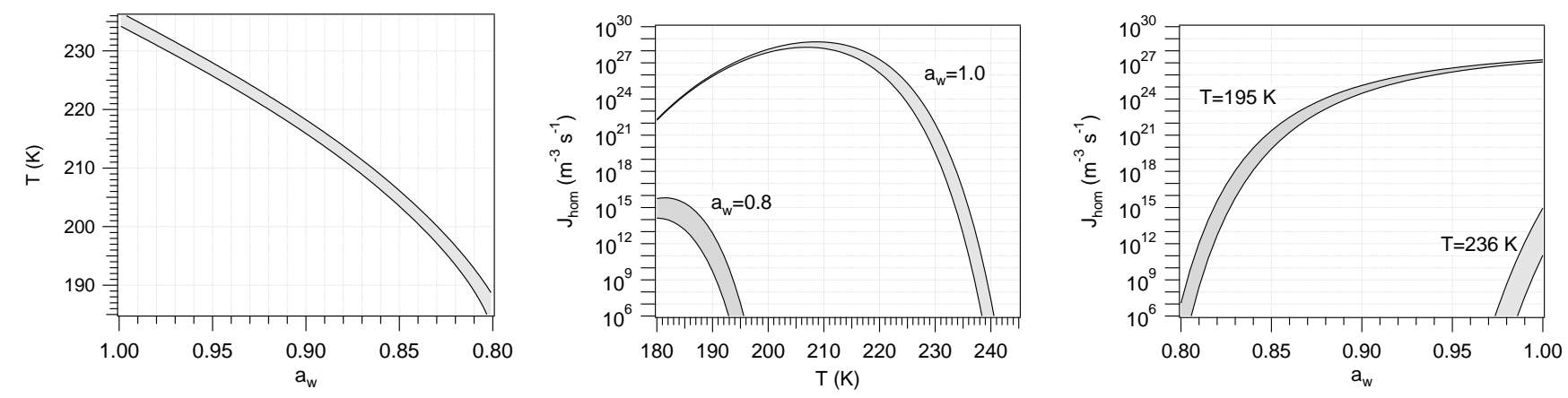

Figure 8. Estimated range of variability in $T_{\mathrm{f}}\left(D_{p}=10 \mu \mathrm{m}\right.$ and $\left.\Delta t=10\right)$ and $J_{\text {hom }}$ for the NNF model.

belong to the interface (which is likely an upper limit of variability) will increase $\Gamma_{\mathrm{w}}$ to 1.51 . The factor, $s$, is 1.09 for hcp crystals and 1.12 for bcc crystals (Jian et al., 2002) and it is not likely that $s$ would be outside of this range. Figure 8 shows the expected variation in $J_{\text {hom }}$ from variation in $\Gamma_{\mathrm{w}}$ and $s$ within these intervals. It represents between 1 and 3 orders of magnitude variation in $J_{\mathrm{hom}}$, and about $2 \mathrm{~K}$ variability in freezing temperatures.

\section{Conclusions}

The model presented here constitutes a new thermodynamic framework for nucleation that does not use the interfacial tension as defining parameter. It is therefore free from bias induced by uncertainties in the parameterization of $\sigma_{\text {iw }}$. Instead, an expression for the interfacial energy was developed from first principles using thermodynamic arguments. The new framework is based on a conceptual model in which the interface is considered to be made of "water molecules trapped by the solid matrix". It also accounts for the finite droplet size leading to changes in the composition of the liquid phase upon nucleation. The proposed framework model is fundamentally different from classical nucleation theory in that it does not consider the curvature of the germ as determinant of nucleation but rather emphasizes the entropic changes across the interface. Since it places emphasis on the increase in order and the reduction in entropy across the interface, the new model has been termed the Negentropic Nucleation Framework, NNF.

Comparison against experimental results showed that the new framework is able to reproduce measured nucleation rates and is capable of explaining the observed constant shift in water activity between melting and nucleation (Koop et al., 2000). The constant water activity shift originates because the freezing temperature only exist for a very narrow range of $\Delta a_{\mathrm{w}}$ (Eq. 37), and represents a balance between kinetic and thermodynamic constraints to nucleation. NNF shows that the effect of water activity on nucleation is a manifestation of the entropic barrier to the formation of the germ. A theoretical expression for $\Delta a_{\mathrm{w}}$ was derived and was shown to agree well with experimental values (Koop et al., 2000; Koop and Zobrist, 2009). This constitutes the first phenomenological derivation of the water activity criteria found by Koop et al. (2000).

The new framework shows that the interfacial energy depends strongly on $a_{\mathrm{w}}$. This dependency originates from the excess concentration of either solute or solvent when the dividing surface is defined. Such excess is present even if the EDS is defined with respect to the solvent. Since $a_{\mathrm{w}}$ is a control variable in nucleation it is advantageous to define the EDS with respect to the solute and explicitly calculate the solvent surface excess. By application of this procedure it was shown that the interfacial energy is a function of water activity only and independent of the nature of the solute.

The origin of the dependency of $J_{\text {hom }}$ on $a_{\mathrm{w}}$ was elucidated by applying several independent expressions for the interfacial tension within the framework of CNT. It was shown that $a_{\mathrm{w}}$ alters $J_{\text {hom }}$ by modification of the surface excess affecting $\sigma_{\text {iw }}$ and by increasing the energy of "unmixing" required to create a solute-free ice germ. Two new expressions were derived to parameterize $\sigma_{\text {iw }}$. The first one uses the NNF model and accounts explicitly for surface excess. By using this expression it was shown that the constant in the classical Turnbull (1950) approximation to $\sigma_{\text {iw }}$ (Eq. 25) can be interpreted as a measure of the thickness of the interfacial layer around the ice germ. The second expression for $\sigma_{\text {iw }}$ was empirically derived by fitting CNT to K00. It was inferred that $\sigma_{\text {iw }}$ derived in this way does not only account for surface effects but also acts a correction factor for the assumption of negligible mixing effects in CNT. Since in CNT $\sigma_{\text {iw }}$ represents surface effects only, it is not clear whether empirically derived expressions for $\sigma_{\mathrm{iw}}$ are consistent with the assumptions of CNT.

Analysis of the new framework suggested that the temperature dependency of $\Delta G_{\text {nuc }}$ and $\Delta G_{\text {act }}$ plays a significant role in defining $J_{\text {hom }}$ and $T_{\mathrm{f}}$. It was shown that around $T_{\mathrm{f}}$ the increase in $\Delta G_{\text {act }}$ as $T$ decreases is balanced by a decrease in $\Delta G_{\text {nuc }}$. Thus an increased driving force for nucleation compensates for the slower molecular diffusion at low $T$. Such coupling between kinetics and thermodynamics 
during nucleation suggests that a thermodynamic description of the pre-exponential factor (Eq. 19) may be possible.

The model presented here emphasizes the entropic nature of homogeneous nucleation. Molecular simulations may shed further light on the role of entropy changes across the interface in ice nucleation. Measurements of the interface thickness would also help elucidate the role of the ice crystal lattice structure and the thickness of the interfacial layer (represented by the constants $s$ and $\Gamma_{\mathrm{w}}$, respectively) in determining $J_{\text {hom }}$.
The framework introduced here reconciles theoretical and experimental results. Since it obviates the usage of $\sigma_{\text {iw }}$ as defining parameter, it may help reducing the uncertainty in $J_{\text {hom }}$ associated with the parameterization of $\sigma_{\text {iw }}$ in theoretical models. The new framework offers for the first time a thermodynamically consistent explanation of the effect of water activity on ice nucleation. Its relative simplicity makes it suitable to describe ice nucleation in cloud models, and may lead to a better understanding of the formation of ice in the atmosphere. 


\section{Appendix A}

Table A1. List of symbols.

\begin{tabular}{|c|c|}
\hline$a_{\mathrm{w}}, a_{y}$ & Activity of water and solute, respectively \\
\hline$a_{\mathrm{w}, \mathrm{eq}}$ & Equilibrium $a_{\mathrm{W}}$ between bulk liquid and ice (Koop and Zobrist, 2009) \\
\hline$D_{p}$ & Droplet diameter, $10 \mu \mathrm{m}$ \\
\hline$f_{\mathrm{f}}$ & Droplet freezing fraction \\
\hline$G$ & Gibbs free energy \\
\hline$h$ & Planck’s constant \\
\hline$h_{\mathrm{W}, \mathrm{s}}, h_{\mathrm{w}, 1 \mathrm{~s}}$ & Partial molar enthalpy of water in bulk ice and in the interface, respectively \\
\hline$J_{0}$ & Pre-exponential factor \\
\hline$J_{\text {hom }}$ & Nucleation rate \\
\hline$k_{\mathrm{B}}$ & Boltzmann constant \\
\hline$n$ & Total number of molecules in the solid germ \\
\hline$n^{*}$ & Critical germ size \\
\hline$n_{\mathrm{s}}, n_{\mathrm{ls}}$ & Number of molecules in the bulk of the solid and in the interface, respectively \\
\hline$N_{\mathrm{c}}$ & Number of atoms in contact with the ice germ, $5.85 \times 10^{18} \mathrm{~m}^{-2}$ (Pruppacher and Klett, 1997) \\
\hline$N_{\mathrm{W}}, N_{y}$ & Total number of water and solute molecules, respectively \\
\hline$p_{\mathrm{s}, \mathrm{w}}, p_{\mathrm{s}, \mathrm{i}}$ & Liquid water and ice saturation vapor pressure, respectively (Murphy and Koop, 2005) \\
\hline$s$ & Geometric constant relating $n$ and $n_{\mathrm{ls}}, 1.105 \mathrm{~mol}^{2 / 3}$ \\
\hline$S_{\mathrm{i}}$ & Saturation ratio with respect to ice \\
\hline$s_{\mathrm{W}, \mathrm{s}}, s_{\mathrm{W}, 1 \mathrm{~s}}$ & Partial molar entropy of water in bulk ice and in the interface, respectively \\
\hline$T$ & Temperature \\
\hline$T_{\mathrm{f}}$ & Freezing temperature \\
\hline$v_{\mathrm{d}}$ & Droplet volume \\
\hline$v_{\mathrm{W}}$ & Molecular volume of water in ice (Zobrist et al., 2007) \\
\hline$Z$ & Zeldovich factor \\
\hline$\Delta G_{\text {act }}$ & Activation energy of liquid water (Zobrist et al., 2007) \\
\hline$\Delta G_{\text {nuc }}$ & Nucleation work \\
\hline$\Delta G_{\text {sln }}$ & Change in free energy of the bulk solution during nucleation \\
\hline$\Delta h_{\mathrm{w}, 1 \mathrm{~s}}$ & Excess enthalpy of the interface \\
\hline$\Delta h_{\mathrm{f}}$ & Heat of fusion of water* \\
\hline$\Delta t$ & Experimental timescale, $10 \mathrm{~s}$ \\
\hline$\Delta a_{\mathrm{W}}$ & $a_{\mathrm{w}}-a_{\mathrm{w}, \mathrm{eq}}$ \\
\hline$\Phi$ & Energy of formation of the interface \\
\hline$\Gamma_{\mathrm{w}}$ & Molecular surface excess of water, 1.46 \\
\hline$\Gamma_{y}$ & Molecular surface excess of solute \\
\hline$\mu_{\mathrm{W}}, \mu_{y}$ & Chemical potential of water and solute, respectively \\
\hline$\mu_{\mathrm{w}, \mathrm{ls}}$ & Chemical potential of water at the interface \\
\hline$\mu_{\mathrm{W}, \mathrm{s}}$ & Chemical potential of bulk ice \\
\hline$\rho_{\mathrm{W}}, \rho_{\mathrm{i}}$ & Bulk density of liquid water and ice, respectively (Pruppacher and Klett, 1997) \\
\hline$\sigma_{\mathrm{iw}}$ & Ice-liquid interfacial energy \\
\hline$\Omega_{\mathrm{g}}$ & Ice germ surface area \\
\hline
\end{tabular}

* From the data of Johari et al. (1994) the following fit was obtained:

$\Delta h_{\mathrm{f}}=7.50856 \times 10^{-7} T^{5}-8.40025 \times 10^{-4} T^{4}+0.367171 T^{3}-78.1467 T^{2}+8117.02 T-3.29032 \times 10^{5}\left(\mathrm{~J} \mathrm{~mol}^{-1}\right)$ for $T$ between 180 and $273 \mathrm{~K}$ 
Acknowledgements. Donifan Barahona was supported by the NASA Modeling, Analysis and Prediction program under WBS 802678.02.17.01.07. The author thanks Athanasios Nenes for his comments on the manuscript.

Edited by: Y. Cheng

\section{References}

Alpert, P. A., Aller, J. Y., and Knopf, D. A.: Initiation of the ice phase by marine biogenic surfaces in supersaturated gas and supercooled aqueous phases, Phys. Chem. Chem. Phys., 13, 19882-19894, 2011.

Asta, M., Beckermann, C., Karma, A., Kurz, W., Napolitano, R., Plapp, M., Purdy, G., Rappaz, M., and Trivedi, R.: Solidification microstructures and solid-state parallels: Recent developments, future directions, Acta Materialia, 57, 941-971, 2009.

Baker, M. and Baker, M.: A new look at homogeneous freezing of water, Geophys. Res. Lett., 31, L19102, doi:10.1029/2004GL0204, 2004.

Barahona, D.: On the ice nucleation spectrum, Atmos. Chem. Phys., 12, 3733-3752, doi:10.5194/acp-12-3733-2012, 2012.

Barahona, D. and Nenes, A.: Dynamical states of low temperature cirrus, Atmos. Chem. Phys., 11, 3757-3771, doi:10.5194/acp11-3757-2011, 2011.

Barahona, D., Rodriguez, J., and Nenes, A.: Sensitivity of the global distribution of cirrus ice crystal concentration to heterogeneous freezing, J. Geophys. Res., 15, D23213, doi:10.1029/2010JD014273, 2010.

Barahona, D., Molod, A., Bacmeister, J., Nenes, A., Gettelman, A., Morrison, H., Phillips, V., and Eichmann, A.: Development of two-moment cloud microphysics for liquid and ice within the NASA Goddard earth observing system model (GEOS-5), Geosci. Model Dev. Discuss., 6, 5289-5373, doi:10.5194/gmdd6-5289-2013, 2013.

Bauerecker, S., Ulbig, P., Buch, V., Vrbka, L., and Jungwirth, P.: Monitoring ice nucleation in pure and salty water via high-speed imaging and computer simulations, J. Phys. Chem. C, 112, 76317636, 2008.

Black, S.: Simulating nucleation of molecular solids, P. Roy. Soc. A, 463, 2799-2811, 2007.

Bourne, J. and Davey, R.: The role of solvent-solute interactions in determining crystal growth mechanisms from solution: I. The surface entropy factor, J. Cryst. Growth, 36, 278-286, 1976.

Brukhno, A. V., Anwar, J., Davidchack, R., and Handel, R.: Challenges in molecular simulation of homogeneous ice nucleation, J. Phys. Cond. Matter, 20, 494243, doi:10.1088/09538984/20/49/494243, 2008.

Bullock, G. and Molinero, V.: Low-density liquid water is the mother of ice: on the relation between mesostructure, thermodynamics and ice crystallization in solutions, Faraday Discuss., doi:10.1039/C3FD00085K, in press, 2013.

Digilov, R. M.: Semi-empirical model for prediction of crystal-melt interfacial tension, Surface Sci., 555, 68-74, 2004.

Dufour, L. and Defay, R.: Thermodynamics of Clouds (International Geophysics Series; Vol. 6), Academic Press, New York, 1963.

Errington, J. R., Debenedetti, P. G., and Torquato, S.: Cooperative origin of low-density domains in liquid water, Phys. Rev. Lett., 89, 215503, 2002.
Ford, I. J.: Properties of ice clusters from an analysis of freezing nucleation, J. Phys. Chem. B, 105, 11649-11655, 2001.

$\mathrm{Fu}, \mathrm{Q}$.: An accurate parameterization of the solar radiative properties of cirrus clouds for climate models, J. Climate, 9, 2058 2082, 1996.

Gettelman, A., Liu, X., Barahona, D., Lohmann, U., and Chen, C.: Climate impacts of ice nucleation, J. Geophys. Res., 117, D20201, doi:10.1029/2012JD017950, 2012.

Gibbs, J. W.: The collected works of J. Willard Gibbs, Yale University Press, Yale, 1957.

Hartmann, D., Holton, J., and Fu, Q.: The heat balance of the tropical tropopause, cirrus, and stratospheric dehydration, Geophys. Res. Lett., 28, 1969-1972, 2001.

Haymet, A. and Oxtoby, D. W.: A molecular theory for the solidliquid interface, J. Chem. Phys., 74, 2559, 1981.

Hiemenz, P. C. and Rajagopalan, R.: Principles of Colloid and Surface Chemistry, revised and expanded, vol. 14, CRC Press, New York, 1997.

Jensen, E. J. and Pfister, L.: Transport and freeze-drying in the tropical tropopause layer, J. Geophys. Res., 109, D02207, doi:10.1029/2003JD004022, 2004.

Jensen, E. J., Toon, O. B., and Hamill, P.: Homogeneous freezing nucleation of stratospheric solution droplets, Geophys. Res. Lett., 18, 1857-1860, 1991.

Jian, Z., Kuribayashi, K., and Jie, W.: Solid-liquid interface energy of metals at melting point and undercooled state, Materials Trans., 43, 721-726, 2002.

Johari, G., Fleissner, G., Hallbrucker, A., and Mayer, E.: Thermodynamic continuity between glassy and normal water, J. Phys. Chem., 98, 4719-4725, 1994.

Jones, D.: The free energies of solid-liquid interfaces, J. Mat. Sci., 9, 1-17, 1974.

Kärcher, B. and Lohmann, U.: A parameterization of cirrus cloud formation: homogeneous freezing including effects or aerosol size, J. Geophys. Res., 107, 4698, doi:10.1029/2001JD001429, 2002.

Karim, O. A. and Haymet, A.: The ice/water interface: A molecular dynamics simulation study, J. Chem. Phys., 89, 6889, 1988.

Kashchiev, D.: Nucleation: basic theory with applications, Butterworth-Heinemann, Oxford, 2000.

Ketcham, W. and Hobbs, P.: An experimental determination of the surface energies of ice, Phil. Mag., 19, 1161-1173, 1969.

Khvorostyanov, V. I. and Curry, J. A.: Thermodynamic theory of freezing and melting of water and aqueous solutions, J. Phys. Chem. A, 108, 11073-11085, 2004.

Khvorostyanov, V. I. and Curry, J.: Critical humidities of homogeneous and heterogeneous ice nucleation: Inferences from extended classical nucleation theory, J. Geophys. Res., 114, D04207, doi:10.1029/2008JD011197, 2009.

Knopf, D. A. and Rigg, Y. J.: Homogeneous ice nucleation from aqueous inorganic/organic particles representative of biomass burning: Water activity, freezing temperatures, nucleation rates, J. Phys. Chem. A, 115, 762-773, 2011.

Koop, T. and Zobrist, B.: Parameterizations for ice nucleation in biological and atmospheric systems, Phys. Chem. Chem. Phys., 11, 10839-10850, 2009.

Koop, T., Luo, B., Tslas, A., and Peter, T.: Water activity as the determinant for homogeneous ice nucleation in aqueous solutions, Nature, 406, 611-614, 2000. 
Laaksonen, A., Talanquer, V., and Oxtoby, D. W.: Nucleation: Measurements, theory, and atmospheric applications, Ann. Rev. Phys. Chem., 46, 489-524, 1995.

Liu, X., Penner, J., Ghan, S., and Wang, M.: Inclusion of ice microphysics in the NCAR Community Atmospheric Model version 3 (CAM3), J. Climate, 20, 4526-4547, 2007.

Lohmann, U. and Feichter, J.: Global indirect aerosol effects: a review, Atmos. Chem. Phys., 5, 715-737, doi:10.5194/acp-5-7152005, 2005.

Lohmann, U. and Kärcher, B.: First interactive simulations of cirrus clouds formed by homogeneous freezing in the ECHAM general circulation model, J. Geophys. Res., 107, 4105, doi:10.1029/2001JD000767, 2002.

MacKenzie, A.: Are the (Solid-Liquid) Kelvin Equation and the Theory of Interfacial Ten sion Components Commensurate?, J. Phys. Chem. B, 101, 1817-1823, 1997.

Malkin, T. L., Murray, B. J., Brukhno, A. V., Anwar, J., and Salzmann, C. G.: Structure of ice crystallized from supercooled water, P. Natl. Acad. Sci. USA, 109, 1041-1045, 2012.

Matsumoto, M., Saito, S., and Ohmine, I.: Molecular dynamics simulation of the ice nucleation and growth process leading to water freezing, Nature, 416, 409-413, 2002.

Moore, E. B. and Molinero, V.: Structural transformation in supercooled water controls the crystallization rate of ice, Nature, 479, 506-508, 2011.

Murphy, D. and Koop, T.: Review of the vapour pressures of ice and supercooled water for atmospheric applications, Q. J. Roy. Meteorol. Soc., 131, 1539-1565, 2005.

Murray, B., Broadley, S., Wilson, T., Bull, S., Wills, R., Christenson, H., and Murray, E.: Kinetics of the homogeneous freezing of water, Phys. Chem. Chem. Phys., 12, 10380-10387, 2010a.

Murray, B., Wilson, T., Dobbie, S., Cui, Z., Al-Jumur, S., Mähler, O., Schnaiter, M., Wagner, R., Benz, S., Niemand, M., Saathoff, H., Ebert, V., Wagner, S., and Kärcher, B.: Heterogeneous nucleation of ice particles on glassy aerosol under cirrus conditions, Nat. Geosci., 3, 233-237, 2010 b.
Pruppacher, H. and Klett, J.: Microphysics of clouds and precipitation, 2nd Edn., Kluwer Academic Publishers, Boston, MA, 1997.

Reinhardt, A. and Doye, J. P.: Note: Homogeneous TIP4P/2005 ice nucleation at low supercooling, J. Chem. Phys., 139, 096102, doi:10.1063/1.4819898, 2013.

Reinhardt, A., Doye, J. P., Noya, E. G., and Vega, C.: Local order parameters for use in driving homogeneous ice nucleation with all-atom models of water, J. Chem. Phys., 137, 194504, 2012.

Riechers, B., Wittbracht, F., Hütten, A., and Koop, T.: The homogeneous ice nucleation rate of water droplets produced in a microfluidic device and the role of temperature uncertainty, Phys. Chem. Chem. Phys., 15, 5873-5887, 2013.

Schay, G.: A comprehensive presentation of the thermodynamics of adsorption excess quantities, Pure Appl. Chem., 48, 393-400, 1976.

Spaepen, F.: A structural model for the solid-liquid interface in monatomic systems, Acta Metallurgica, 23, 729-743, 1975.

Spaepen, F.: Homogeneous nucleation and the temperature dependence of the crystal-melt interfacial tension, Solid State Phys., 47, 1-32, 1994.

Tabazadeh, A., Jensen, E., and Toon, O.: A model description for cirrus cloud nucleation from homogeneous freezing of sulfate aerosols, J. Geophys. Res., 102, 23485-23850, 1997.

Turnbull, D.: Formation of crystal nuclei in liquid metals, J. App. Phys., 21, 1022-1028, 1950.

Wu, D. T., Gránásy, L., and Spaepen, F.: Nucleation and the SolidLiquid Interfacial Free Energy, MRS Bull., 29, 945-950, 2004.

Zobrist, B., Koop, T., Luo, B., Marcolli, C., and Peter, T.: Heterogeneous ice nucleation rate coefficient of water droplets coated by a nonadecanol monolayer, J. Phys. Chem. C, 111, 2149-2155, 2007.

Zobrist, B., Marcolli, C., Peter, T., and Koop, T.: Heterogeneous ice nucleation in aqueous solutions: the role of water activity, J. Phys. Chem. A, 112, 3965-3975, 2008. 